\title{
Jerk Analysis of a Power-Split Hybrid Electric Vehicle Based on a Data-Driven Vehicle Dynamics Model
}

\author{
Xiaohua Zeng ${ }^{1}$, Haoyong Cui ${ }^{1}$, Dafeng Song ${ }^{1, *}$, Nannan Yang ${ }^{1}$, Tong Liu ${ }^{1}$, Huiyong Chen ${ }^{2}$, \\ Yinshu Wang ${ }^{2}$ and Yulong Lei ${ }^{1}$ \\ 1 State Key Laboratory of Automotive Simulation and Control, Jilin University, Changchun 130025, China; \\ zeng.xiaohua@126.com (X.Z.); cuihy16@mails.jlu.edu.cn (H.C.); 15543019521@163.com (N.Y.); \\ liutong242@163.com (T.L.); chyzsq@163.com (Y.L.) \\ 2 Zhengzhou Yutong Bus Co., Ltd., Zhengzhou 450016, China; 18844198446@163.com (H.C.); \\ 18844196995@163.com (Y.W.) \\ * Correspondence: songdf@126.com; Tel.: +86-0431-85094866
}

Received: 25 April 2018; Accepted: 8 June 2018; Published: 13 June 2018

\begin{abstract}
Given its highly coupled multi-power sources with diverse dynamic response characteristics, the mode transition process of a power-split Hybrid Electric Vehicle (HEV) can easily lead to unanticipated passenger-felt jerks. Moreover, difficulties in parameter estimation, especially power-source dynamic torque estimation, result in new challenges for jerk reduction. These two aspects entangle with each other and constitute a complicated coupling problem which obstructs the realization of a valid anti-jerk method. In this study, a vehicle dynamics model with reference to a data-driven modeling method is first established, integrating a full-time artificial neural network engine dynamic model that can accurately predict engine dynamic torque. Then the essential reason for the occurrence of vehicle jerks in real driving conditions is analyzed. Finally, to smooth the mode transition process, a more practical anti-jerk strategy based on power-source torque changing rate limitation (TCRL) is proposed. Verification studies indicate that the data-driven vehicle dynamics model has enough accuracy to reflect the vehicle dynamic characteristics, and the proposed TCRL strategy could reduce the vehicle jerk by up to $85.8 \%$, without any sacrifice of vehicle performance. This research provides a feasible method for precise modeling of vehicle dynamics and a reference for improving the riding comfort of hybrid electric vehicles.
\end{abstract}

Keywords: hybrid electric vehicle; data-driven modeling method; real vehicle data; torque changing rate limitation; anti-jerk strategy; riding comfort

\section{Introduction}

Due to the demand of low fuel consumption and environment-friendly exhaust emissions, the development of hybrid electric vehicles (HEVs) and electric vehicles (EVs) has further accelerated in recent years. EVs (especially fuel cell EVs) are considered to be the best potential solutions for future transportation [1], while HEVs are an essential transition between EVs and conventional internal combustion engine vehicles.

The powertrain configuration of an HEV can be divided into three types: series, parallel and power-split [1]. Among the various proposed HEV powertrains, power-split HEV is considered to be one of the most promising configurations. Typical power-split HEVs possess one or more planetary gear sets along with one engine and two motors. Engine output power is split into two paths (mechanical and electric) by the planetary gear mechanism. As for motors, one is used predominantly as a generator, while the other is used predominantly as a traction motor and occasionally used as a generator during regenerative braking (RB). Due to the dual-degree-freedom characteristic of planetary 
gear sets, the engine speed and torque in power-split hybrid electric powertrains can be freely adjusted by dual motors, making it possible for the engine to operate at the most efficient areas and helping power-split HEVs exhibit better fuel economy compared with other HEV configurations [2].

In the field of HEV research, existing studies mostly specified fuel economy as the primary research objective [3,4], and several have focused on the emission characteristics of HEVs [5,6]. However, both the fuel economy and emission characteristic of HEVs are associated with relatively low-bandwidth nature, while the higher bandwidth nature, vehicle riding comfort, is often ignored by most existing HEV strategies [7]. As an essential vehicle performance index, the riding comfort of HEVs also deserves special consideration [8].

The riding comfort of conventional vehicles involves two aspects: the NVH characteristic and the vehicle jerk problem (which is caused by improper control during clutch engagement and disengagement). Both of these aspects are also applicable to evaluating the riding comfort of HEVs. Nevertheless, many other riding comfort problems peculiar to HEVs arise because of an additional application of the motor.

On the one hand, several studies have already focused on HEVs' NVH issues. Takayoshi et al. explored certain NV problems peculiar to HEVs, such as vehicle, engine, motor/generator vibration and noise, and improved the NVH characteristics of a Toyota Prius by improving its mechanical structure and optimizing the control strategy [9]. Zou et al. investigated the vibration and noise characteristics of a power-split planetary hybrid transmission by establishing a planetary mechanism dynamic model and produced a numerical analysis method of distinctive natural frequencies and corresponding vibration modes [10].

On the other hand, vehicle jerk is common during the mode transition process of HEVs due to miscoordination between power sources, thereby significantly affecting riding comfort. Many studies have focused on the vehicle jerk problem of HEVs [11]. Koprubasi et al. designed a mode transition control approach that is based on the state-space methodology to smooth the variation rate of vehicle acceleration and thus achieve a seamless mode transition process for parallel HEVs [12]. Beck et al. proposed a model predictive control strategy of a parallel HEV to achieve a smooth mode transition process without driving power interruption and sensible jerks [13]. The abovementioned studies mostly focused on the parallel hybrid system, which consists of a single motor only besides the engine, and mainly used the PID and feedback control methodology to overcome vehicle jerk [14]. As for power-split hybrid electric systems, which comprise dual motors and an engine, jerk is considerably more pronounced because the three power sources are highly coupled and their dynamic response characteristics are diverse [15]. Applying coordination control strategies to prevent jerk in power-split HEVs is common [16]. Wang et al. designed a coordinated control strategy of a compound power-split hybrid vehicle, adopting active damping control and carrier torque estimation to suppress engine ripple torque during engine cranking [17]. Zhu et al. adopted a fuzzy gain-scheduling PID controller as feedback to compensate for the various nonlinearities of power-split systems and further enhance vehicle drivability and performance [18]. In [19], we proposed a dynamic coordination control strategy that is based on a model prediction methodology to improve the riding comfort of a dual planetary hybrid electric system [19]. However, all these anti-jerk methods are simulation-oriented and can thus provide a reference for real-vehicle anti-jerk control to a certain extent, but cannot be used directly in real vehicles because of the required time-consuming calculations. Therefore, a more practical control strategy is needed to solve the jerk problems of power-split HEVs under real driving conditions.

Before the presentation of a specific anti-jerk method, a vehicle dynamics model of the power-split $\mathrm{HEV}$ is needed to reproduce jerks that occur in real driving conditions and provide a test environment to verify the effectiveness of the proposed control strategy further. Simplified vehicle dynamics models are widely adopted in current HEV jerk research because establishing a precise vehicle dynamics model is difficult [20]. A simplified parallel HEV model was established as the basis of the proposed mode-transition-control approach in [12], and quadratic terms related to velocity are neglected in the vehicle dynamics model in [13] to reduce the difficulty of controller design. Certain studies on vehicle 
jerk problems have adopted a simple first-order-lag engine model to predict engine torque $[13,18,19]$. These simplifications would detract from the accuracy of vehicle dynamics models and result in inaccuracies in model parameter estimation, especially power-source dynamic torque estimation, which is key to limiting the control effect of most proposed anti-jerk strategies. By contrast, data-driven modeling methods have considerably progressed in recent years because of its independence from the internal mechanism of modeling objects. Numerous studies regarding data-driven modeling are available in the field of battery capacity estimation [21,22]. Data-driven modeling also has applications in vehicle dynamics modeling. Sun et al. presented a torque coordination strategy that is based on data-driven predictive control to decrease vehicle jerk during mode transition, but the modeling data are obtained from simulation. Thus, the established model may lack authenticity [23].

Power-source dynamic torque estimation is an essential aspect of vehicle dynamics modeling, and the establishment of engine dynamic models is considered as an example herein to illustrate the advantages of data-driven modeling. There is difficulty in creating an accurate engine model that can fully reflect engines' real-time dynamic characteristics because of the inherent high nonlinearity and time-delay characteristics of engines [24]. The engine steady-state model and the engine dynamic model are widely adopted in current HEV simulation. The quasi-static engine torque obtained from the engine steady-state model cannot satisfy the requirements of dynamic calculation in jerk simulations [25]. Engine dynamic models include the mean torque engine model, crankshaft transient torque engine model, and artificial neural network (ANN) engine model which can reflect engine dynamic characteristics to varying degrees [26]. The mean torque engine model and the crankshaft transient torque engine model yield a large deviation when predicting high engine operating loads because they depend on the simulation of complex engine intake processes [27]. As a typical representative of data-driven modeling method, ANN can approximate any complex nonlinear relationship and is widely used in engine dynamic modeling [28,29]. Accuracy of the ANN engine model can be guaranteed because such model avoids specific details of engine intake and combustion processes [30]. However, traditional ANN engine dynamics models (AEDMs) mostly focus on the normal operating dynamic characteristics of engines regardless of the engine starting and stopping processes occurring frequently in HEVs under real driving conditions, which are crucial for the generation of vehicle jerks [31].

The present study focuses on the riding comfort problem of HEVs and specifically the vehicle longitudinal jerk problem occurring in real driving conditions of a dual planetary power-split hybrid electric bus (HEB). The original contributions of this paper are as follows: (1) An exact vehicle dynamics model based on the data-driven modeling method is established, which provides an innovative solution for vehicle dynamic modeling. The established data-driven vehicle model can effectively represent the complex dynamic characteristics of the system and provide a valid and reliable computing environment for the system's optimal control without the considerable calculations and a large number of parameters required by the traditional mechanism model; (2) A practical anti-jerk strategy based on power-source torque changing rate limitation (TCRL) is proposed. This strategy can calculate the threshold of power-source torque changing rate in real time according to the system's complex as well as time-varying constraints and adapt well to the complex driving conditions of real vehicles. Compared with more complex mode transition strategies, the TCRL has a smaller amount of calculations and fully meets the requirements of online applications.

The remaining sections of this study are organized as follows: in Section 2, the configuration of the dual planetary power-split HEB is introduced, and a system dynamics analysis is conducted to obtain the essence of vehicle jerk. In Section 3, the modeling process of full-time AEDM based on real vehicle data is introduced. In Section 4, the integrated vehicle dynamics model is established, and its accuracy is validated. In Section 5, the reasons for vehicle jerks that occur in real drive cycles are summarized, and the corresponding solution (power-source TCRL strategy) is proposed. Moreover, the effectiveness of the proposed strategy is validated by simulation. In Section 6, the conclusions and remarks are presented. 


\section{System Dynamics Analysis}

\subsection{System Description}

The investigated dual planetary power-split HEB adopts two planetary gear sets as its power-coupling mechanism, as shown in Figure 1.

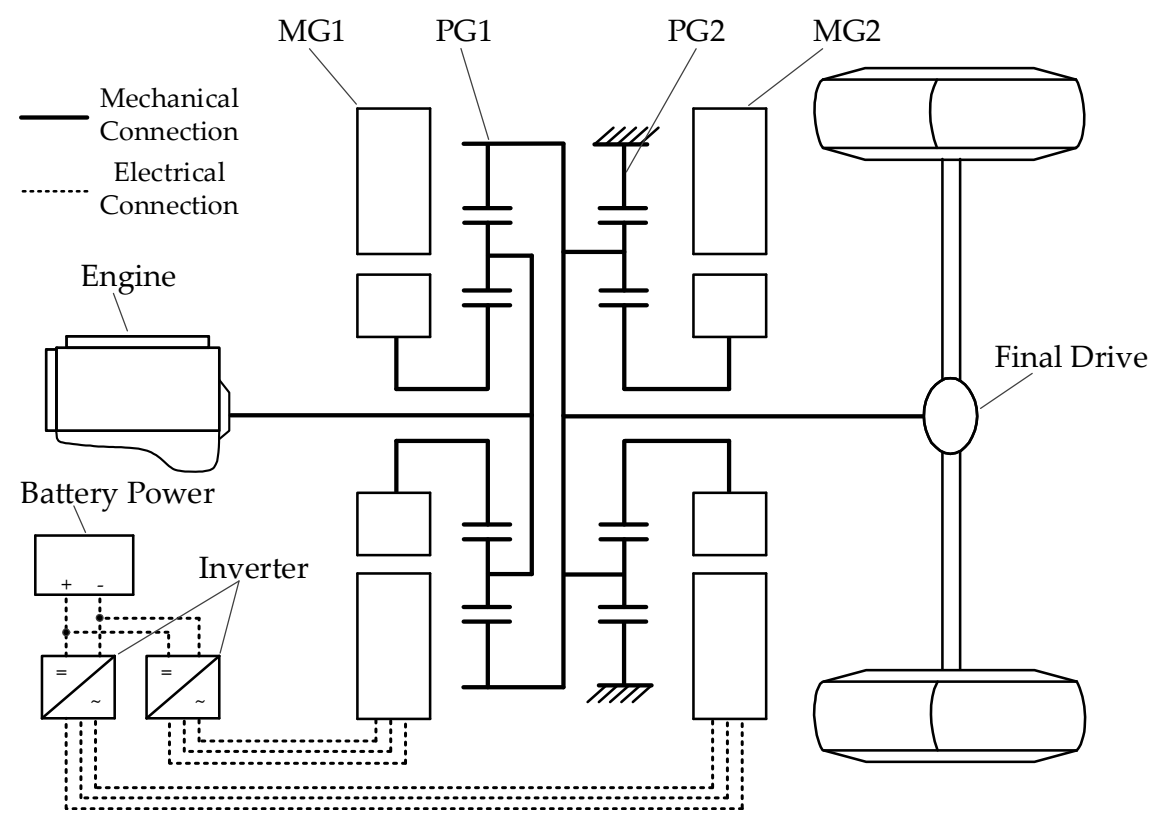

Figure 1. Powertrain of dual planetary power-split hybrid electric bus (HEB).

Specifications of the dual planetary power-split HEB are measured through tests and experiments, and are listed in Table 1.

Table 1. Vehicle specifications.

\begin{tabular}{ccc}
\hline Parameter & Description & Value \\
\hline$m$ & Gross vehicle mass & $12,000 \mathrm{~kg}$ \\
$R_{t}$ & Wheel radius & $0.51 \mathrm{~m}^{2}$ \\
$C_{D}$ & Air drag coefficient & 0.4 \\
$A$ & Vehicle frontal area & $9.0 \mathrm{~m}^{2}$ \\
$i_{0}$ & Final drive ratio & 4.88 \\
$g$ & Gravitation acceleration & $9.81 \mathrm{~m} / \mathrm{s}^{2}$ \\
$k_{1}$ & PG1 characteristic parameter & 2.63 \\
$k_{2}$ & PG2 characteristic parameter & 2.11 \\
$I_{e}$ & Moment of inertia of engine & $1.35 \mathrm{~kg} \cdot \mathrm{m}^{2}$ \\
$I_{g}$ & Moment of inertia of MG1 & $0.33 \mathrm{~kg} \cdot \mathrm{m}^{2}$ \\
$I_{m}$ & Moment of inertia of MG2 & $0.33 \mathrm{~kg} \cdot \mathrm{m}^{2}$ \\
$I_{r}$ & Moment of inertia of ring gear & $0.0015 \mathrm{~kg} \cdot \mathrm{m}^{2}$ \\
$I_{S}$ & Moment of inertia of sun gear & $0.0015 \mathrm{~kg} \cdot \mathrm{m}^{2}$ \\
$I_{c}$ & Moment of inertia of planetary carrier & $0.0015 \mathrm{~kg} \cdot \mathrm{m}^{2}$ \\
\hline
\end{tabular}

Figure 1 shows that the investigated power-split HEB has three power sources, namely, an engine, MG1, and MG2. Both MG1 and MG2 can function as either a generator or a motor alternatively. MG1 serves as a motor that accelerates the engine when engine starts and switches to a generator immediately after the engine has started. MG2 can serve as a motor to supply power to propel the vehicle and as a generator to recover kinetic energy in RB mode. Therefore, the operating conditions of MG1 and MG2 vary frequently, and their characteristics are totally different. 
The front planetary gear set (PG1) of the power-coupling mechanism is the power-split device of the HEB and the rear planetary gear set (PG2) is used as a reducer of MG2 because the ring gear of PG2 is rigidly connected to the transmission house. PG1 influences the power-split and efficiency characteristics of the powertrain, and PG2 only adjusts MG2's speed and torque.

\subsection{Dynamics Analysis}

The powertrain of the dual planetary power-split HEB can be divided into three independent subsystems: PG1, PG2, and the final drive (FD). The internal dynamics of the powertrain is analyzed separately on the basis of these subsystems. Results show that the viscous damping of rotation, the elasticity between bearings and mounts, and the meshing elasticity of gears yield only a negligible difference in the powertrain dynamics analysis; thus, all of them are ignored for simplicity [32].

The free-body diagram of PG1 is illustrated in Figure 2. The equilibrium equations of PG1 are written as:

$$
\begin{gathered}
I_{r 1} \dot{\omega}_{r 1}=F_{1} \cdot R_{1}-T_{r 1} \\
\left(I_{e}+I_{c 1}\right) \dot{\omega}_{e}=T_{e}-F_{1} \cdot R_{1}-F_{1} \cdot S_{1} \\
\left(I_{g}+I_{s 1}\right) \dot{\omega}_{g}=F_{1} \cdot S_{1}-T_{g}
\end{gathered}
$$

where $T, I$ and $\omega$ denote torque $(\mathrm{N} \cdot \mathrm{m})$, moment of inertia $\left(\mathrm{kg} \cdot \mathrm{m}^{2}\right)$ and rotational speed $(\mathrm{rad} / \mathrm{s})$, respectively. As for the subscripts: $r 1, c 1$ and $s 1$ denote the front ring gear, planetary carrier and sun gear, respectively; $e$ the engine; $g$ is for MG1. $R_{1}$ and $S_{1}$ are the radii $(\mathrm{m})$ of the front ring gear, and sun gear, respectively. $F_{1}$ is the internal force $(\mathrm{N})$ of PG1. We specify left as the positive direction of torques.

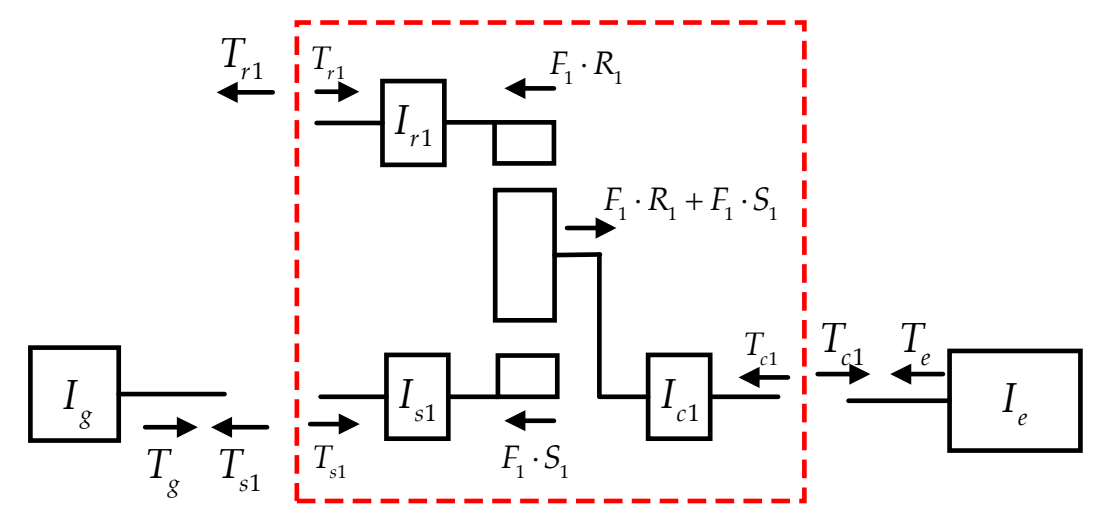

Figure 2. Free-body diagram of PG1.

The free-body diagram of PG2 is illustrated in Figure 3. The equilibrium equations of PG2 are written as:

$$
\begin{gathered}
\left(I_{m}+I_{s 2}\right) \dot{\omega}_{m}=T_{m}-F_{2} \cdot S_{2} \\
I_{c 2} \dot{\omega}_{c 2}=F_{2} \cdot S_{2}+F_{2} \cdot R_{2}-T_{c 2}
\end{gathered}
$$

where subscripts $c 2$ and $s 2$ denote the rear planetary carrier, and sun gear, respectively; $m$ stands for MG2. $R_{2}$ and $S_{2}$ represent the radii $(\mathrm{m})$ of the rear ring gear and sun gear, respectively. $F_{2}$ is the internal force $(\mathrm{N})$ of PG2. 


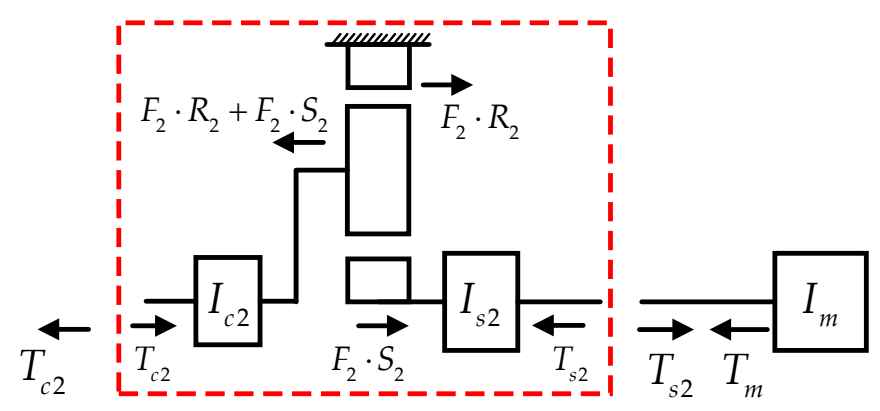

Figure 3. Free-body diagram of PG2.

The relationships between the rotational speeds of the components of PG1 and PG2 are:

$$
\begin{gathered}
\left(1+k_{1}\right) \omega_{c 1}=\omega_{s 1}+k_{1} \omega_{r 1} \\
\left(1+k_{2}\right) \omega_{c 2}=\omega_{s 2}
\end{gathered}
$$

where $k_{1}$ and $k_{2}$ denote the characteristic parameter of PG1 and PG2 respectively, i.e., the ratio of ring gear teeth to sun gear teeth.

According to Equations (4), (5) and (7), Equation (8) is available:

$$
T_{c 2}=T_{m}\left(1+k_{2}\right)-\left[\left(I_{m}+I_{s 2}\right)\left(1+k_{2}\right)^{2}+I_{c 2}\right] \dot{\omega}_{c 2}
$$

The free-body diagram of FD is illustrated in Figure 4. The output torques of PG1 and PG2 are coupled in FD and are transmitted to the wheels to overcome the vehicle driving resistance. The following equation is yielded only when vehicle longitude dynamics is considered and wheel slip is neglected:

$$
\left(\dot{\omega}_{r 1} / i_{o}\right) R_{t}^{2} m=\left(T_{c 2}+T_{r 1}\right) i_{o}-T_{f}
$$

where $i_{0}$ denotes the final drive ratio, $R_{t}$ denotes the wheel radius $(\mathrm{m}), m$ denotes the gross vehicle mass $(\mathrm{kg})$ and $T_{f}$ denotes the vehicle driving resistance torque $(\mathrm{N} \cdot \mathrm{m})$. Ignoring vehicle acceleration resistance and slope resistance, the following relationships exist:

$$
\begin{gathered}
T_{f}=T_{f b}+T_{r}+T_{w} \\
T_{r}=m g f_{r} R_{t} \\
T_{w}=0.5 \rho A C_{D}\left(\omega_{r 1} / i_{o}\right)^{2} R_{t}{ }^{3}
\end{gathered}
$$

where $T_{f b}$ is the braking torque $(\mathrm{N} \cdot \mathrm{m}), T_{r}$ is the rolling resistance torque $(\mathrm{N} \cdot \mathrm{m}), T_{\omega}$ is the aerodynamic drag torque $(\mathrm{N} \cdot \mathrm{m}), f_{r}$ is the rolling resistance coefficient and is a function of vehicle velocity, $A$ denotes the vehicle frontal area $\left(\mathrm{m}^{2}\right)$ and $C_{D}$ denotes the air drag coefficient.

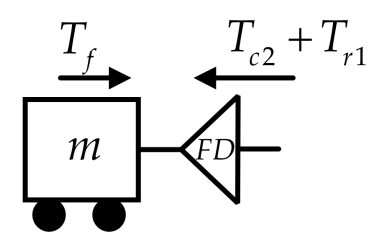

Figure 4. Free-body diagram of final drive (FD).

According to Equations (1), (8)-(12) and the rigid connection of front ring gear and rear planetary carrier, the following relationship can be obtained: 


$$
\begin{gathered}
\dot{\omega}_{r 1}\left[R_{t}^{2} m / i_{o}+I_{r 1} i_{o}+\left(I_{m}+I_{s 2}\right)\left(1+k_{2}\right)^{2} i_{o}+I_{c 2} i_{o}\right] \\
=\left[T_{m}\left(1+k_{2}\right)+F_{1} R_{1}\right] i_{o}-T_{f b}-m g f_{r} R_{t}-0.5 \rho A C_{D}\left(\omega_{r 1} / i_{o}\right)^{2} R_{t}{ }^{3}
\end{gathered}
$$

Finally, the complete dynamics description of the investigated dual planetary power-split system is formed with Equations (2), (3), (6) and (13).

\subsection{Jerk Analysis}

The precise definition of vehicle jerk is the derivative of the vehicle longitudinal acceleration $(a)$, as shown below:

$$
j=\frac{d a}{d t}=\frac{d^{2} v}{d t^{2}}
$$

where $v$ is vehicle velocity $(\mathrm{m} / \mathrm{s})$, which can be expressed as $v=\omega_{r 1} R_{t} / i_{0}$, and Equation (14) is then reformed as Equation (15):

$$
j=\ddot{\omega}_{r 1} R_{t} / i_{o}
$$

Thus, the intensity of vehicle jerk can use $\ddot{\omega}_{r 1}$ to characterize, and the vehicle jerk has the same direction as $\ddot{\omega}_{r 1}$.

According to Equations (2), (3), (6) and (13), the following equation can be obtained:

$$
\begin{array}{r}
\ddot{\omega}_{r 1}\left[\frac{I_{v}^{\prime}}{k_{1} i_{i_{0}} I_{g}^{\prime}}+\frac{\left(1+1 / k_{1}\right)^{2} I_{v}^{\prime}}{i_{0} I_{e}^{\prime}}+1\right]=-\frac{1}{k_{1} I_{g}^{\prime}} \dot{T}_{g}+\left(1+k_{2}\right)\left[\frac{\left(1+1 / k_{1}\right)^{2}}{I_{e}^{\prime}}+\frac{1}{k_{1}^{2} I_{g}^{\prime}}\right] \dot{T}_{m} \\
+\frac{1+1 / k_{1}}{I_{e}^{\prime}} \dot{T}_{e}-\left[\frac{\left(1+1 / k_{1}\right)^{2}}{i_{0} I_{e}^{\prime}}+\frac{1}{k_{1}^{2} i_{o} I_{g}^{\prime}}\right] \dot{T}_{f}
\end{array}
$$

where:

$$
\begin{gathered}
I_{v}^{\prime}=R_{t}{ }^{2} m / i_{0}+4 I_{t} / i_{0}+I_{r 1} i_{o}+\left(I_{m}+I_{s 2}\right)\left(1+k_{2}\right)^{2} i_{o}+I_{c 2} i_{o} \\
I_{e}^{\prime}=I_{e}+I_{c 1} \\
I_{g}^{\prime}=I_{s 1}+I_{g}
\end{gathered}
$$

The coefficient of $\ddot{\omega}_{r 1}$ on the left side of Equation (16) is constant, so jerk $\ddot{\omega}_{r 1}$ is determined only by the four items on the right side of Equation (16). The fourth item on the right side of Equation (16) is related to the torque changing rate of driving resistance. As mentioned in Section 1, the vehicle jerk usually occurs during mode transition processes. Such processes have a short duration and the investigated vehicle is a large inertia system. Hence, the change in vehicle velocity can be ignored. According to Equations (11) and (12), the velocity-dependent items, rolling resistance torque $T_{r}$ and aerodynamic drag torque $T_{\omega}$ will not change either. $T_{f b}$ will not change abruptly as well. Therefore, the driving resistance torque $T_{f}$ approximates a constant and has a small and negligible derivative in the mode transition process. Therefore, system jerk depends mainly on the first three items on the right side of Equation (16).

The first three items on the right side of Equation (16) are of the same form, i.e., the absolute torque changing rate of each power source (i.e., $\dot{T}_{g}, \dot{T}_{m}, \dot{T}_{e}$ ) multiplied by a constant coefficient. This pattern indicates that the jerk of power-split HEVs depends only on the torque changing rate of the three power sources. To normalize the specific contribution of each power source to the vehicle jerk, the concept of relative torque changing rate $U_{1}, U_{2}$, and $U_{3}$ is proposed: three power sources' absolute torque changing rates $\dot{T}_{g}, \dot{T}_{m}$, and $\dot{T}_{e}$ multiplied by corresponding powertrain-parameter-related coefficient, as shown in Equations (17)-(19):

$$
\begin{gathered}
U_{1}=-\frac{1}{k_{1} I_{g}^{\prime}} \dot{T}_{g} \\
U_{2}=\left(1+k_{2}\right)\left[\frac{\left(1+1 / k_{1}\right)^{2}}{I_{e}^{\prime}}+\frac{1}{k_{1}^{2} I_{g}^{\prime}}\right] \dot{T}_{m}
\end{gathered}
$$




$$
U_{3}=\frac{1+1 / k_{1}}{I_{e}^{\prime}} \dot{T}_{e}
$$

\section{Full-time Artificial Neural Network Engine Dynamic Model}

A vehicle dynamics model integrating an engine dynamic model should be established prior to developing an effective anti-jerk control strategy. However, obtaining the exact value of engine output torque is difficult due to the nonlinear behavior of engine subsystems, especially in real driving conditions, where the operating conditions of the engine change frequently [33]. The establishment of an engine dynamic model, which is the strongest nonlinear component in power-split hybrid system, is the most important aspect of vehicle dynamics modeling. According to data-driven modeling theory, this section proposes to establish a full-time AEDM with reference to the ANN modeling method by utilizing the engine operating data acquired from the real drive cycles of the investigated dual planetary power-split HEB.

\subsection{Artificial Neural Network Introduction}

In the field of ANN engine modeling, the back propagation (BP) neural network is the most commonly used ANN because of its simple structure [34]. This study adopts a BP neural network to establish the full-time AEDM. A BP neural network consists of several layers of neurons, including input layer, output layer, and hidden layers, as shown in Figure 5. The evaluation indexes of BP neural network accuracy are the mean square error (MSE) and the correlation coefficient (R). The smaller the MSE and the larger the R (maximum 1) mean, the smaller the error between the output value of the $\mathrm{ANN}$ and the desired value.

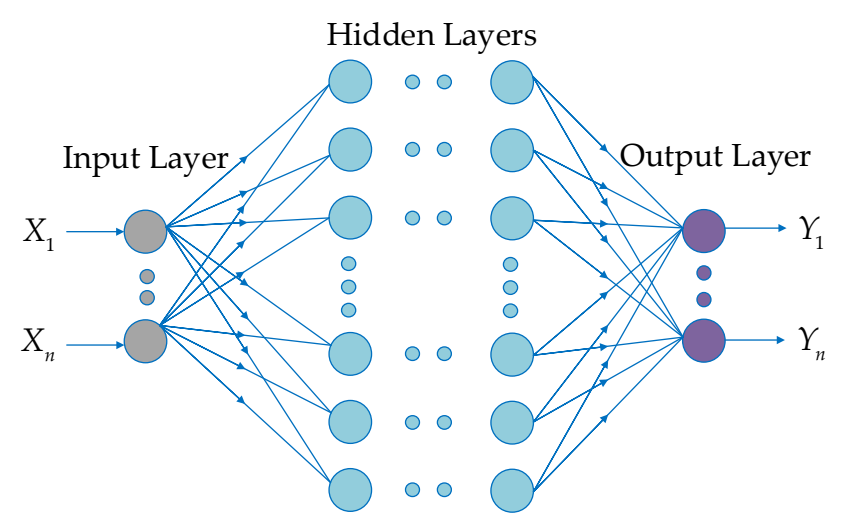

Figure 5. Structure of back propagation (BP) neural network.

\subsection{Engine Dynamic Model Establishment}

\subsubsection{Training Sample Data Selection}

Training sample data should be selected before the training of the ANN engine dynamic model. According to Equation (16), engine real-time torque is needed to calculate vehicle jerk $\ddot{\omega}_{r 1}$. Thus, engine torque data obtained from real driving conditions are used as the ANN desired variable, and the obtained engine speed and fuel rate data are adopted as ANN input variables. A series of drive cycle tests focused on the investigated power-split HEB is executed on a straight and flat road and marked Cycle 1, Cycle 2, and so on. Each cycle comprises several similar driving parts. A part of Cycle 1, marked Cycle 1 Part 1, is selected as the training sample data of ANN. The values of vehicle velocity and engine speed, engine fuel rate, and engine output torque of Cycle 1 Part 1, are presented in Figure 6. 


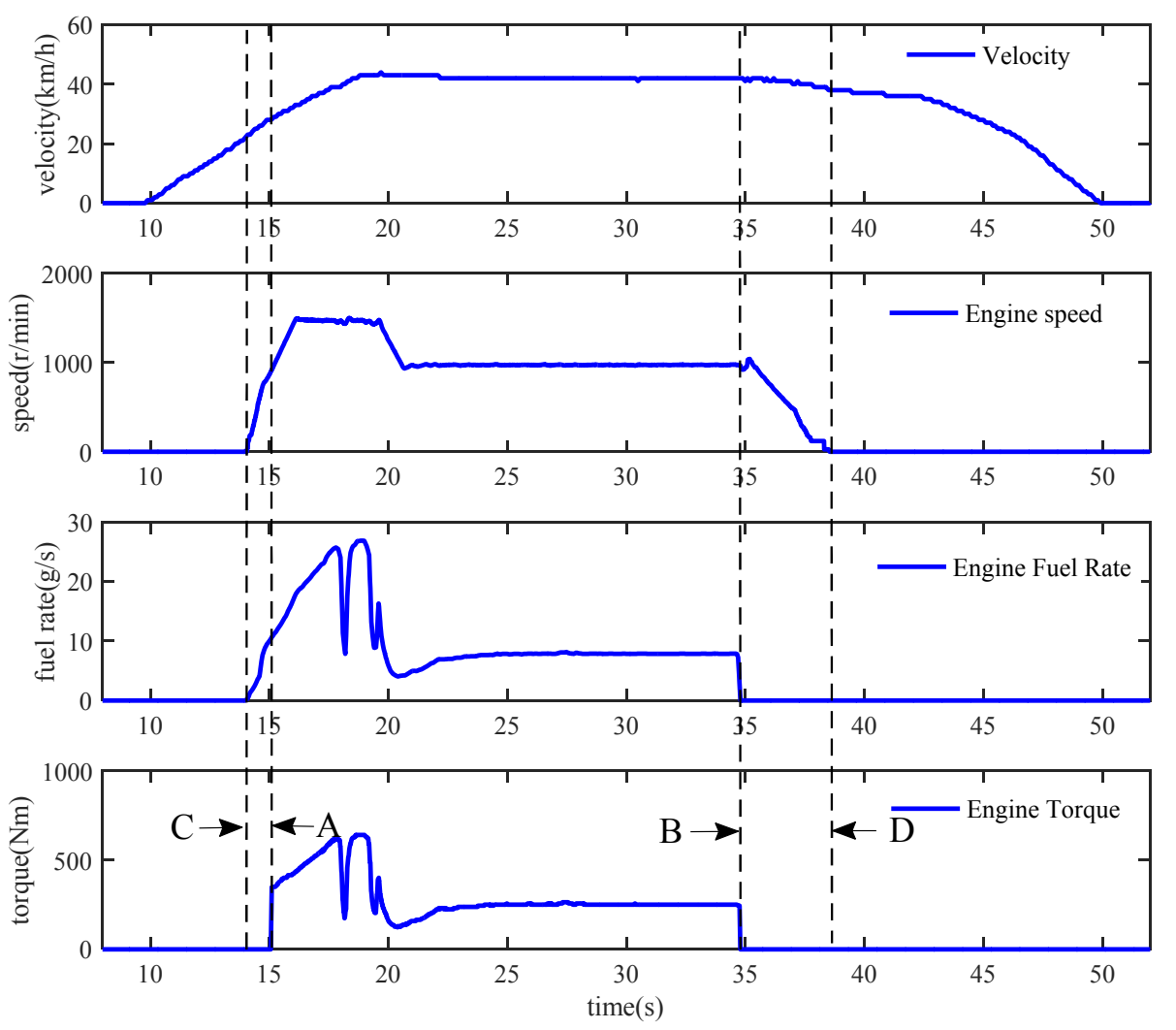

Figure 6. Data of Cycle 1 Part 1.

According to the trend of velocity in Figure 6, Cycle 1 Part 1 of the investigated dual planetary power-split HEB can be divided into the starting condition, acceleration condition, constant velocity condition, braking condition, and parking condition. Furthermore, on the basis of the operating mode of the investigated power-split HEB, the above operating conditions can be further subdivided. From $10 \mathrm{~s}$ to A in Figure 6, the power-split HEB operates in electrical vehicle (EV) mode, and the vehicle, driven solely by MG2, launches and accelerates. From $C$ to $20 \mathrm{~s}$ in Figure 6, the power-split HEB operates at electronic variable transmission (EVT) sport (EVT-S) mode, where the engine, together with MG2, work at a heavy load with large torque changing rates to meet the driver's accelerating request. From $20 \mathrm{~s}$ to B in Figure 6, the power-split HEB operates at EVT economic (EVT-E) mode, where the engine and MG2 are in a relatively stable state, with both torques relatively low. From $B$ to $50 \mathrm{~s}$ in Figure 6, the power-split HEB operates at RB mode, where the engine stops and MG2 works at generating condition to recover vehicle kinetic energy, and the vehicle decelerates and stops eventually.

Meanwhile, for the engine, Figure 6 shows that Cycle 1 Part 1 contains driving phases of engine torque soaring, engine torque slumping, and engine torque relatively stable state. For general ANN engine modeling, the engine operating data of these phases are sufficient because they cover the various conditions that an engine can encounter. The demand torque of the investigated power-split HEB varies widely due to the uncertainty of vehicle driving conditions. Thus, the engine starts and stops frequently because of the switching of operating modes. Therefore, the engine starting and stopping processes are particularly important for power-split system engine modeling. To establish an engine dynamic model that can fully reflect engine real-time dynamic characteristics, this study proposes an improved ANN engine modeling method called the full-time AEDM from the aspect of practical application, with emphasis on engine starting and stopping characteristics. Not only the engine normal operating phase in Cycle 1 Part 1 (AB segment in Figure 6) but also the engine starting phase (CA segment in Figure 6) and stopping phase (BD segment in Figure 6) are adopted to train the ANN engine dynamic model. 
The selected training sample data of the ANN engine model in Cycle 1 Part 1 include all driving conditions of the investigated vehicle, all operating modes of the dual planetary power-split HEB, and the various working conditions of the engine. Hence, the selected Cycle 1 Part 1 is representative for the training of the ANN engine dynamic model.

\subsubsection{Artificial Neural Network Structure}

After training sample data selected, the ANN structure should be determined. This step is the key to BP neural network design. The ANN structure contains two aspects: the number of hidden layers and the number of neurons in each hidden layer. A BP neural network has two hidden layers at most, and a single-hidden-layer BP neural network can map all continuous functions [35]. When an engine starts and stops, the engine torque changes sharply but does not exhibit a strict step function. Thus, a single-hidden-layer BP neural network is sufficient to meet the engine modeling accuracy requirements.

The number of neurons in each hidden layer is determined by the values of MSE and R. The variation profiles of MSE and R under different hidden neurons are shown in Figure 7.

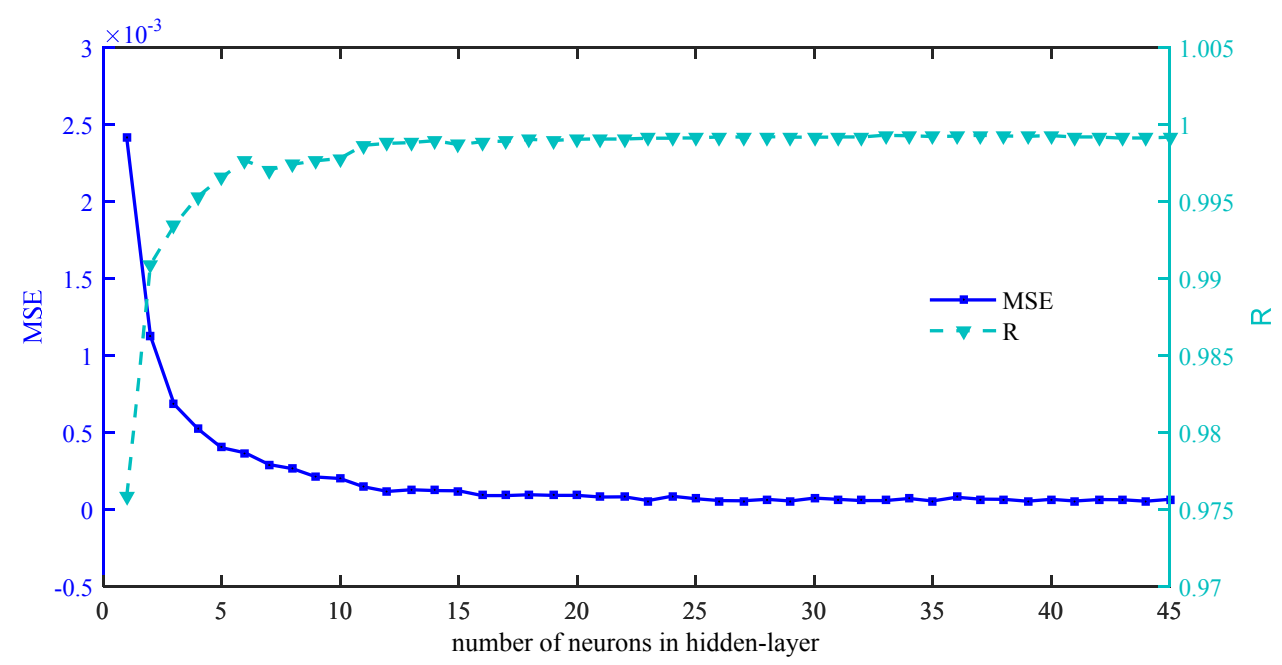

Figure 7. Variation trend of mean square error (MSE) and R of neural network.

As shown in Figure 7, MSE decreases from the initial $2.5 \times 10^{-3}$ and converges to approximately $5 \times 10^{-5}$ with an increase in the number of neurons, and $R$ increases from the initial 0.975 and converges to a final value of approximately 0.999 . A small MSE and an approaching- $1 R$ indicate that the accuracy of the trained ANN engine dynamic model is within the acceptable range. According to ANN theory, the more neurons are contained in the hidden layer, the more accurate the trained ANN model but the longer the training time. With a comprehensive consideration of model accuracy and training time, an ANN with a single hidden layer and 35 neurons is determined. Corresponding MSE and $R$ are $5.904 \times 10^{-5}$ and 0.9993 , respectively.

In conclusion, the ANN structure comprises three layers: an input layer, an output layer, and a hidden layer. The number of neurons in the input and output layers are 2 and 1, respectively, corresponding to the number of input and output variables, and the number of neurons in the hidden layer is set as 35 .

\subsubsection{Artificial Neural Network Generalization Capability Verification}

After the ANN is trained, its generalization capability should be verified. This capability refers to the capacity of the trained ANN to identify data other than training sample data [22]. In terms of the differences between drive cycles, we divide the generalization capability of the trained full-time AEDM into two aspects: local and global generalization capabilities. 
Local generalization capability refers to the capacity of the trained full-time AEDM to fit actual engine torques of Cycle 1 Parts (other than Part 1) in response to the similarity of the velocity trend and power-source operating conditions among the Parts of Cycle 1. Here, Cycle 1 Part 5 and Cycle 1 Part 9 are used as examples to conduct a comparison between the output torque of the full-time AEDM and the actual engine torque, as shown in Figure $8 \mathrm{a}, \mathrm{b}$.

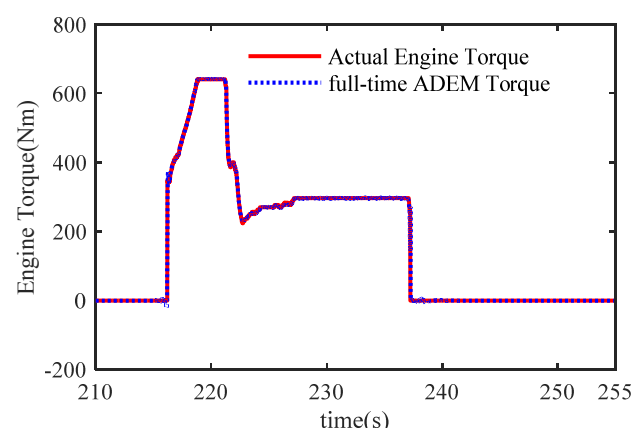

(a)

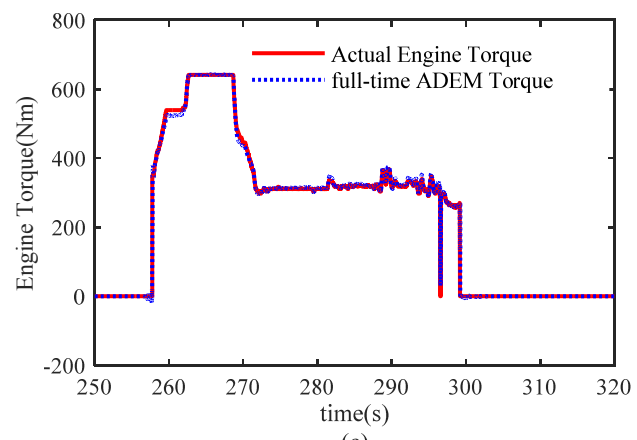

(c)

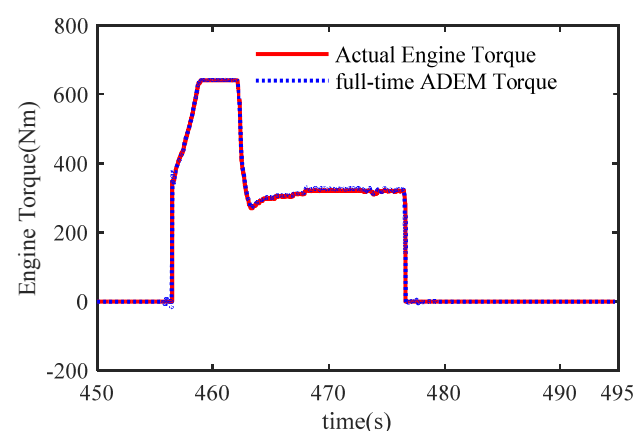

(b)

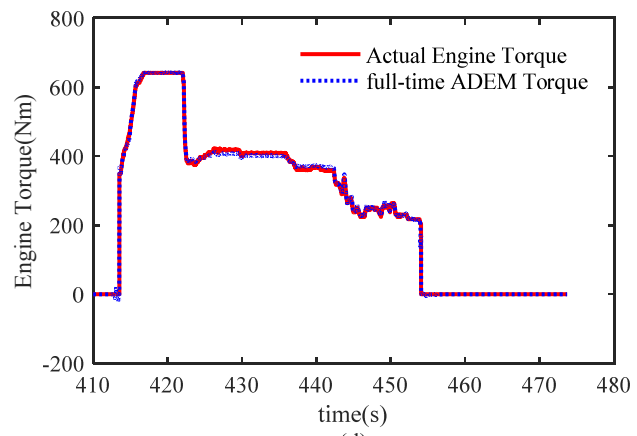

(d)

Figure 8. Torques comparison: (a) Cycle 1 Part 5; (b) Cycle 1 Part 9; (c) Cycle 2 Part 4; (d) Cycle 3 Part 6.

These figures show that the full-time AEDM output torques agree reasonably well with the actual engine torques in Cycle 1 Part 5 and Cycle 1 Part 9. The full-time AEDM output torque follows the actual engine torque accurately in the dynamic and quasi-steady states, where torques change abruptly and smoothly, respectively. The trained full-time AEDM has good local generalization capability and can accurately predict the real-time engine torques of Cycle 1.

Global generalization capability refers to the capacity of the trained full-time AEDM to fit actual engine torques of the Parts of the other cycles in response to the difference in velocity trend and power-source operating conditions among cycles. Only the comparison results between the full-time AEDM output torques and actual engine torques in Cycle 2 Part 4 and Cycle 3 Part 6 are shown due to space limitations (Figure $8 \mathrm{c}, \mathrm{d}$ ). The full-time AEDM can fully predict the real-time torque of the engine beyond Cycle 1 with certain small discrepancies. Therefore, the trained full-time AEDM has good global generalization capability.

The maximum error between the full-time AEDM output torques and the actual engine torques of all Cycles are shown in Table 2, which indicates that the maximum error between the two torques of any Cycle is no more than $\pm 6 \%$ and is within the acceptable range. Therefore, the trained full-time AEDM based on real vehicle data has good generalization capability and can fully reflect the engine real-time dynamic characteristics regardless of driving condition. The establishment of the full-time AEDM lays a foundation for establishing an accurate vehicle dynamics model. 
Table 2. Maximum error between full-time ANN engine dynamics model (AEDM) output torque and actual engine torque.

\begin{tabular}{cc}
\hline Cycle & Maximum Error (\%) \\
\hline Cycle 1 & 4.71 \\
Cycle 2 & -3.75 \\
Cycle 3 & -2.77 \\
Cycle 4 & -2.32 \\
Cycle 5 & -5.01 \\
Cycle 6 & -2.75 \\
\hline
\end{tabular}

\section{Integration and Verification of Data-driven Vehicle Dynamics Model}

With the consideration of vehicle driving resistance, a data-driven dual planetary power-split HEB dynamics model integrating an engine dynamic model and dual motor models is established in MATLAB/Simulink (R2015b, The MathWorks, Inc, Natick, MA, USA). This model serves as a testing environment for the designed control strategy, which is presented in a subsequent section. Furthermore, verification research is conducted to guarantee the accuracy of the established vehicle dynamics model and ensure the credibility of the dynamics analysis in Section 2.

\subsection{Integration of Data-Driven Vehicle Dynamics Model}

An accurate engine dynamic model, the full-time AEDM, has been established in Section 3. According to the vehicle dynamics equation (Equation (16)), dual motor models and a vehicle driving resistance model should be established to obtain a complete vehicle dynamics model.

\subsubsection{Dual Motor Models}

First, models for MG1 and MG2 are established. Simplified motor models are adopted for two reasons. On the one hand, the moment of inertia of the motor shaft is smaller than that of the engine crankshaft. Thus, the motor has rapid response capability and can therefore respond to the demand torque nearly without any time delay. On the other hand, unlike the engine, which possesses high nonlinearities resulting from inconsistent combustion processes, the motor easily achieves precise control with good linearity. These factors jointly contribute to the ideal dynamic characteristics of the motor. Hence, simplified motor models are sufficient for general vehicle dynamics modeling. Similar to previous works, this study introduces a look-up table motor model [1], which regards motor rotational speed and load signal as input and motor torque as output.

\subsubsection{Vehicle Driving Resistance Model}

A vehicle driving resistance model is established. As derived in Equation (10), three separate parts form the vehicle driving resistance torque $T_{f}$ : the braking torque $T_{f b}$, the rolling resistance torque $T_{r}$, and the aerodynamic drag torque $T_{\omega}$.

The total braking torque of the dual planetary power-split HEB can be divided into two parts: regenerative braking torque and mechanical braking torque. It should be pointed out that the braking torque $T_{f b}$ in Equation (10) represents mechanical braking torque only and is proportional to the brake cylinder pressure $P$. When a vehicle operates at RB mode, MG2 serves as a generator to recover vehicle kinetic energy and $T_{m}$ represents the regenerative braking torque.

The rolling resistance torque is calculated from the rolling resistance coefficient $f$, which is related to vehicle velocity and obtained from a look-up table. The aerodynamic drag torque is also a velocity-dependent item and calculated from another look-up table. 


\subsubsection{Vehicle Model Integration}

By integrating the full-time AEDM, dual motor models and vehicle driving resistance model, the data-driven vehicle dynamics model is established in MATLAB/Simulink. For convenient model accuracy verification and jerk calculation, an extended simulation model consisting of an input block, a controller block, the data-driven vehicle dynamics model, and an output block is further established. The top-level structure of the extended simulation model is depicted in Figure 9. The controller block here represents the original control strategy (without anti-jerk function); its detailed description is not covered by the scope of this article.

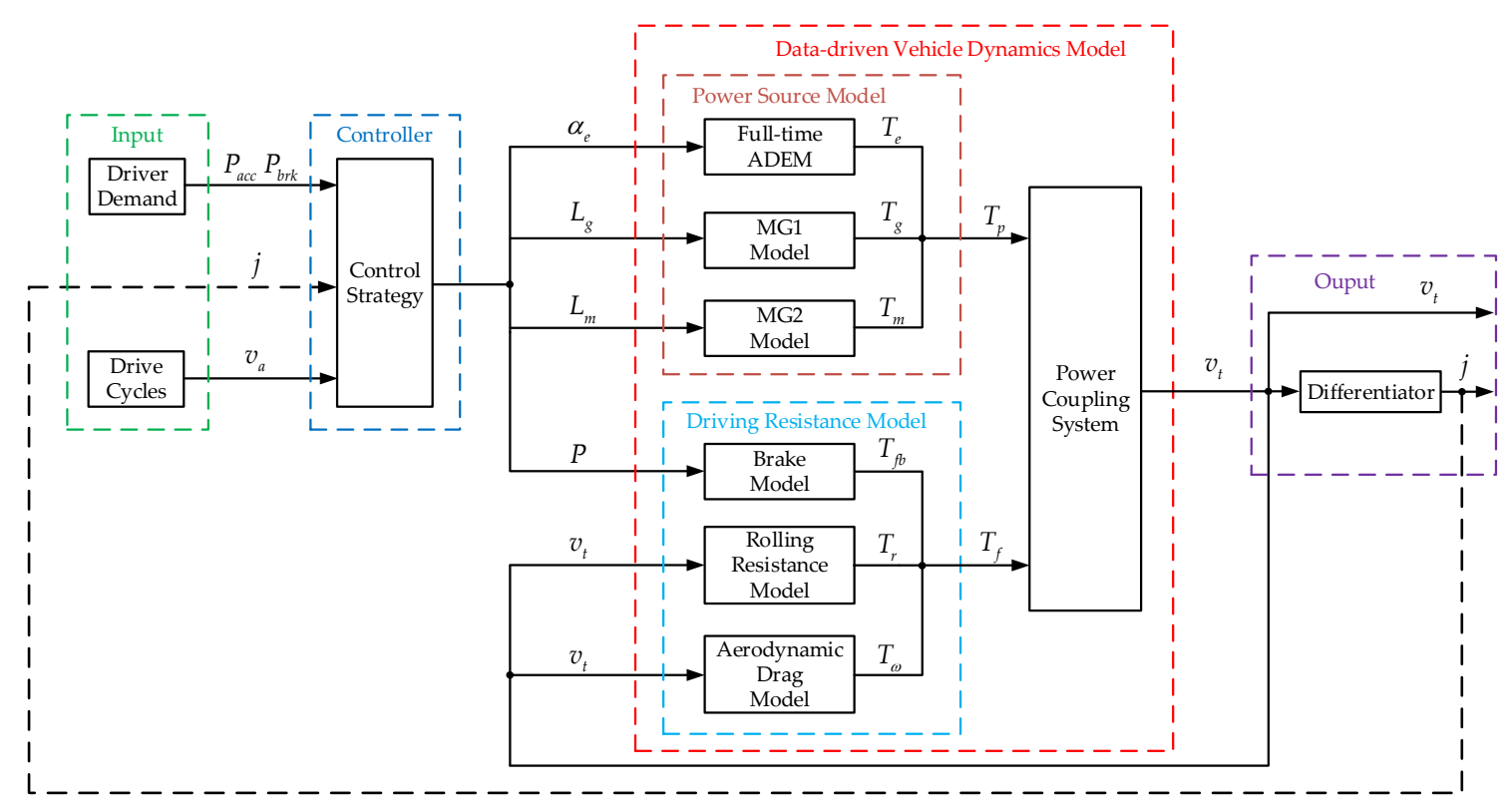

Figure 9. Top-level of extended simulation model.

As shown in Figure 9, the extended simulation model regards driver's accelerator pedal data $P_{a c c}$, driver's brake pedal data $P_{b r k}$, and actual vehicle velocity data $v_{a}$ as the model input because the original control strategy solves the driver's demand torque on the basis of these signals. After calculation and processing, the original control strategy generates load signals of the three power sources and transforms them to corresponding power source model to meet the driver's demand. Load signals work with corresponding power-source rotational speeds to obtain the output torque of each power source. The original control strategy also generates the pressure of brake cylinder $P$ and transforms it to the brake model to obtain the desired mechanical braking torque.

The output torques of the power sources are accumulated to form vehicle propulsive torque $T_{p}$, while the mechanical braking torque, the rolling resistance torque, and aerodynamic drag torque are accumulated to form the vehicle driving resistance torque $T_{f}$. Propulsive torque and resistance torque are coupled in power coupling system and act on the vehicle to obtain theoretical vehicle velocity $v_{t}$. The calculated $v_{t}$ is further transformed to the vehicle driving resistance model to calculate the velocity-dependent items, i.e., the rolling resistance torque and aerodynamic drag torque. According to Equation (16), vehicle jerk is the second derivative of vehicle velocity with respect to time. Thus, a differentiator exists at the end of the extended simulation model. This differentiator regards the theoretical vehicle velocity as input and exports the vehicle jerk.

\subsection{Model Accuracy Verification}

Verification research is conducted to guarantee the accuracy of the established vehicle dynamics model. As mentioned above, when actual driver demand data $P_{a c c}, P_{b r k}$ and actual vehicle velocity 
data $v_{a}$ are regarded as the input of the extended simulation model, the data-driven vehicle dynamics model exports theoretical velocity $v_{t}$. Model accuracy verification is conducted on the basis of the comparison between theoretical velocity $v_{t}$ and actual velocity $v_{a}$. Any drive cycle can be applied to conduct model verification research because the established models have good adaptabilities. For this verification research, Cycle 1 Part 4 is selected. The comparison results are depicted in Figure 10.

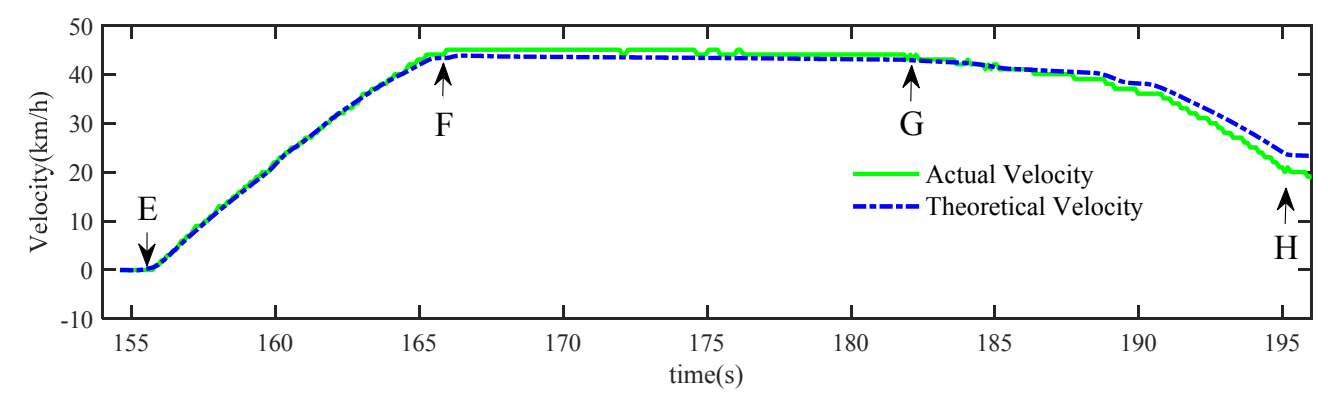

Figure 10. Comparison between theoretical velocity and actual velocity of Cycle 1 Part 4 .

As shown in Figure 10, Cycle 1 Part 4 can be divided into three consecutive driving phases according to the trend of vehicle velocity: the EF acceleration phase, the FG uniform-velocity phase, and the GH braking phase. In the EF phase, the theoretical velocity can follow the actual velocity nearly without any deviation. In the FG and GH phases, the trend of theoretical velocity is consistent with actual velocity, accompanied with some insignificant and acceptable discrepancies. Inevitable error in the actual velocity measurement and possible inaccuracy in the estimation of rolling resistance and air resistance all likely contribute to uncertain deviation between the theoretical velocity and the actual velocity in the FG and GH phases.

In summary, regardless of driving conditions, the theoretical velocity calculated from the vehicle dynamics model coincides well with the actual velocity. Hence, the modeling process of powertrain components is reasonable, and the accuracy of the established data-driven power-split HEB dynamics model is sufficient for reflecting the driveline dynamics in real driving conditions. The verification results also ensure the credibility of dynamics analysis conducted in Section 2.

\section{Vehicle Jerk Analysis and Improvement}

Vehicle jerk of the investigated power-split HEB occurring in real drive cycles is calculated in this section on the basis of the established and validated data-driven vehicle dynamics model. Reasons for intense vehicle jerks are analyzed as well. Furthermore, a practical control strategy based on TCRL is proposed to smooth jerks, and the effectiveness of the proposed TCRL strategy is quantitatively verified.

\subsection{Vehicle Jerk Calculation}

Prior to detailed jerk analysis, intense jerks occurring in real drive cycles under the original control strategy are calculated. The established vehicle dynamics model can reflect driveline dynamics well. Thus, the vehicle jerk calculated by this model should be precise. The driver pedal data $P_{a c c}, P_{b r k}$ and drive cycle data $v_{a}$, which are obtained from real drive cycles, are used as the input of the extended simulation model. Then, the jerk vehicle encountered in real driving conditions is obtained.

Cycle 1 Part 4 is still adopted here as an example to calculate the vehicle jerk because the calculation process of any drive cycle is similar. The calculated vehicle jerk of Cycle 1 Part 4 is plotted in Figure 11a. 


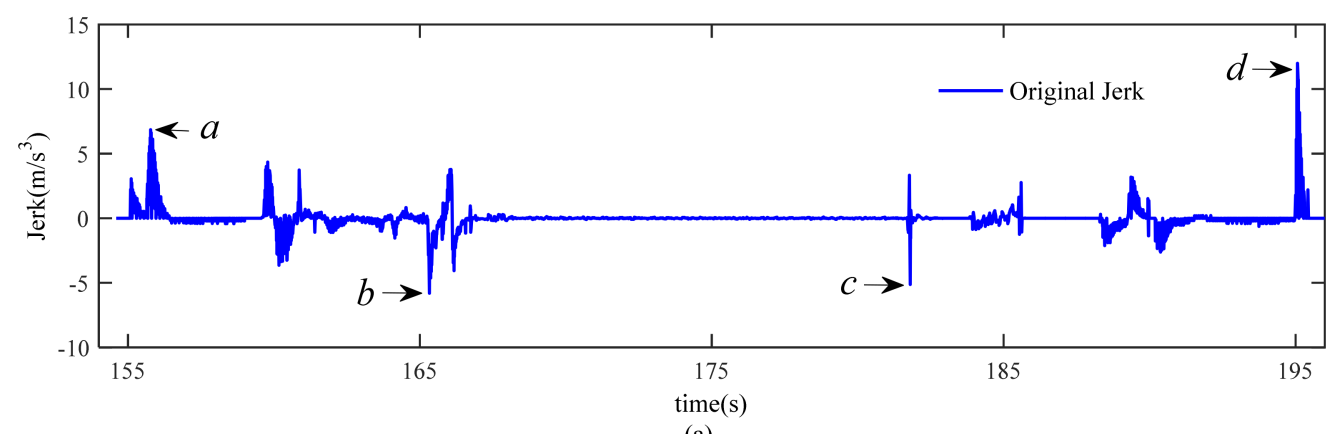

(a)

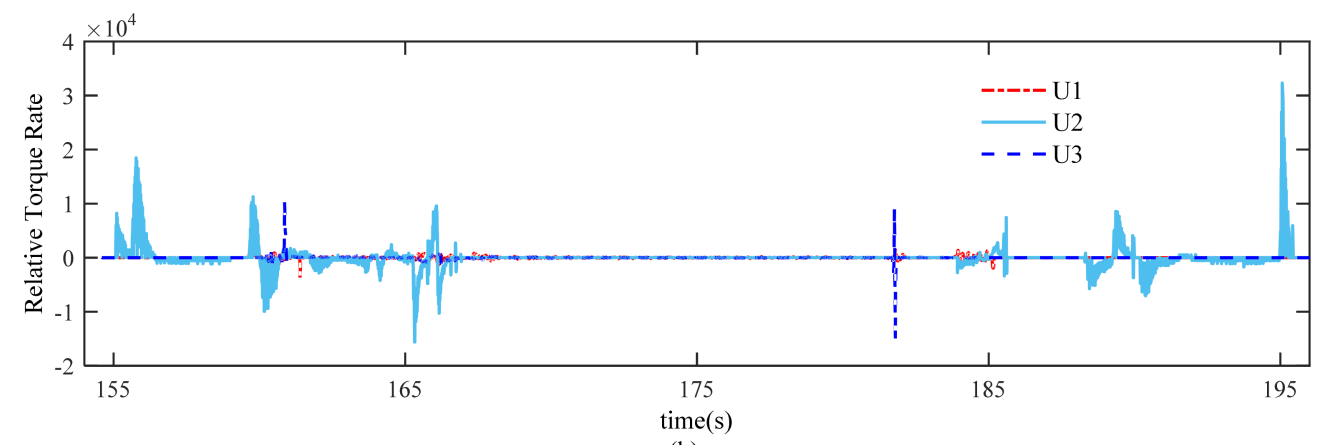

(b)

Figure 11. (a) Vehicle jerk of Cycle 1 Part 4; (b) Relative torque changing rate of Cycle 1 Part 4.

The vehicle jerk in Cycle 1 Part 4 exhibits irregular oscillations, and several driving points have relatively large jerk intensities. The quantitative index of the vehicle jerk varies in different countries; for example, the recommended jerk thresholds in Germany and China are 10 and $17.64 \mathrm{~m} / \mathrm{s}^{3}$, respectively [36]. According to our tests of the investigated power-split HEB, once the absolute intensity of jerk exceeds $5 \mathrm{~m} / \mathrm{s}^{3}$, the jerk becomes noticeable and passengers suffer from poor riding comfort. It is obvious from Figure 11a that a total 4 driving points in Cycle 1 Part 4 where the absolute intensity of vehicle jerks are more than $5 \mathrm{~m} / \mathrm{s}^{3}$. These points are called peak-jerk points and serve as the focus of a detailed analysis of the vehicle jerk.

\subsection{Jerk Detailed Analysis}

As analyzed in Section 2.3, the intensity of vehicle jerk mainly depends on the torque changing rates of three power sources. To distinguish which power source plays a major role in generating jerk, the relative torque changing rates of MG1, MG2 and the engine of Cycle 1 Part 4 are plotted in Figure 11b. Same as the vehicle jerk in Cycle 1 Part 4, the relative torque changing rates of the three power sources exhibit a trend of irregular oscillation. When a peak jerk occurred, there corresponding exists a large relative torque changing rate of a certain power source.

The first peak-jerk point in Cycle 1 Part 4 is taken as an example to conduct a detailed jerk analysis. As shown in Figure 11a, the first peak-jerk point in Cycle 1 Part 4 occurs at $155.8 \mathrm{~s}$, and the intensity of jerk is $6.851 \mathrm{~m} / \mathrm{s}^{3}$ with the positive direction. A partial diagram of the output torques and the relative torque changing rates of the three power sources around the first peak-jerk point is depicted in Figure 12a,b. According to the velocity trend of Cycle 1 Part 4 in Figure 10, the investigated power-split system works at EV mode to launch the vehicle when the first peak jerk occurred. The output torque of MG2 increases drastically to satisfy the driver's launch demand, thereby generating a large relative torque changing rate of MG2 of up to $1.847 \times 10^{4}$. According to Equation (16), a large $U_{2}$ leads to a large positive vehicle jerk. 


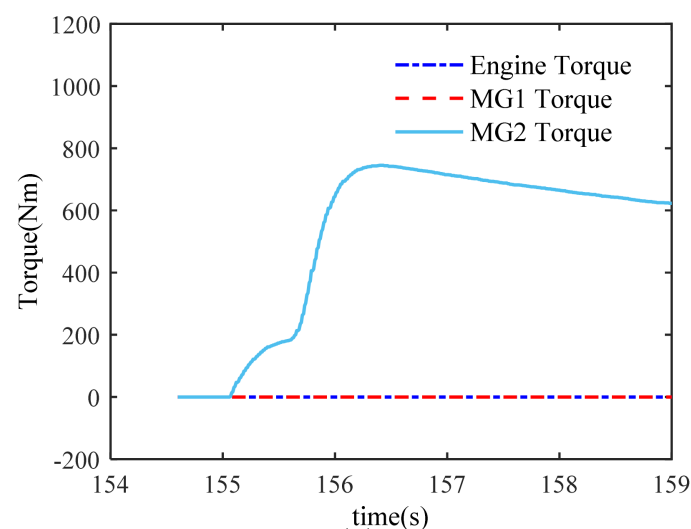

(a)

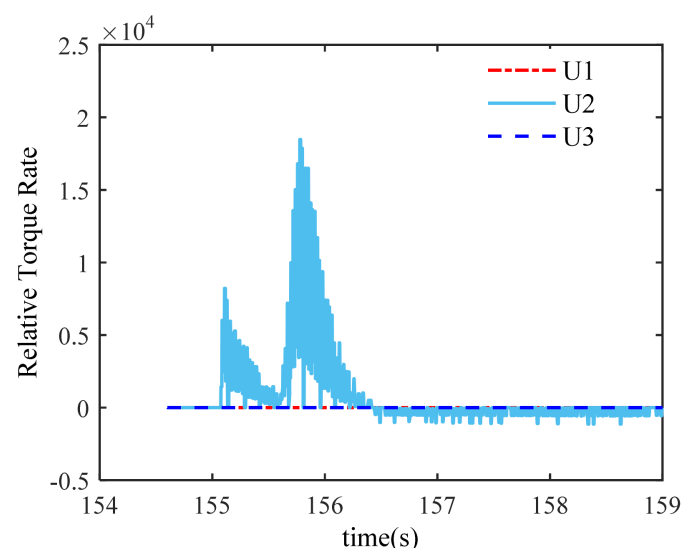

(b)

Figure 12. (a) Torques of power sources around first peak-jerk point of Cycle 1 Part 4; (b) Relative torque changing rates around first peak-jerk point of Cycle 1 Part 4.

Applying the same analysis method to the three other peak-jerk points of Cycle 1 Part 4, the maximum relative torque changing rates of the three power sources during the peak jerks are summarized in Table 3, and the reason for each peak-jerk point is summarized.

Table 3. Reasons for each peak-jerk point of Cycle 1 Part 4.

\begin{tabular}{|c|c|c|c|}
\hline $\begin{array}{l}\text { Peak-Jerk } \\
\text { Number }\end{array}$ & $\begin{array}{l}\text { Intensity } \\
\left(\mathrm{m} / \mathrm{s}^{3}\right)\end{array}$ & $\begin{array}{l}\text { Maximum Relative } \\
\text { Torque Variation Rate }\end{array}$ & Reason for Each Peak-Jerk Point \\
\hline$a$ & 6.851 & $1.847 \times 10^{4}(\mathrm{MG} 2)$ & At EV launch mode, MG2 torque increases rapidly \\
\hline$b$ & -5.806 & $-1.56 \times 10^{4}(\mathrm{MG} 2)$ & $\begin{array}{l}\text { EVT-S mode switches to EVT-E mode, MG2 torque } \\
\text { declines rapidly }\end{array}$ \\
\hline$c$ & -5.133 & $-1.54 \times 10^{4}$ (Engine) & $\begin{array}{l}\text { EVT-E mode switches to RB mode, engine stops with } \\
\text { torque declining rapidly }\end{array}$ \\
\hline$d$ & 11.99 & $3.23 \times 10^{4}(\mathrm{MG} 2)$ & $\begin{array}{l}\text { Exiting RB mode, MG2 stops with torque } \\
\text { declining rapidly }\end{array}$ \\
\hline
\end{tabular}

The launch process of the investigated power-split HEB can be treated as a mode transition process that switches from standstill to EV mode. Hence, it can be concluded from Table 3 that all four peak-jerk points in Cycle 1 Part 4 occur during mode transition process, including the transition from standstill to EV mode, from EVT-S mode to EVT-E mode, from EVT-E mode to RB mode, and the exit of RB mode. The reason for this phenomenon is that mode transition processes of power-split HEVs always accompany the startup or shutdown or sharp torque change of power sources. Once the intervention and withdrawal of a power source lack reasonable and effective control, the power source would easily generate excessive torque changing rate and then unanticipated and intense vehicle jerks would occur. That is exactly most power-split HEV control strategies, including our original control strategy not take into account.

\subsection{Anti-Jerk Method Based on Torque Changing Rate Limitation}

The main reason for intense vehicle jerks occurring under real driving conditions of the investigated power-split HEB is that the output torques of power sources change sharply during the mode transition process. Therefore, the original control strategy of the power-split HEB is improved and a practical anti-jerk strategy based on TCRL is proposed, i.e., with the premise of meeting vehicle performance requirements, reasonably limiting and controlling the torque changing rates of power sources to prevent sharp torque changes. 
Modifying the original control strategy of real power-split HEBs in a short period is difficult. Therefore, to validate the anti-jerk effectiveness of the improved control strategy, the proposed TCRL strategy is first applied to the established data-driven vehicle dynamics model, providing a reference for further improvement of real-vehicle control strategies.

The modification is mainly concentrated on the controller block in Figure 9. The calculated vehicle jerk is fed back to the controller block. Once the detected intensity of jerk exceeds a reasonable threshold, the TCRL strategy is activated and restricts the power-source torque changing rate to the specified target corridor. Cycle 1 Part 4 is still adopted herein to validate the effectiveness of the proposed TCRL strategy. The relative torque changing rates of the three power sources after TCRL are illustrated in Figure 13a. Although irregular oscillation still exists, the maximum relative torque changing rates where peak jerk occurred are significantly lower than those in Figure 11b. The vehicle jerk of Cycle 1 Part 4 after TCRL is shown in Figure 13b, which depicts a dramatic decrease in the intensities of all 4 peak jerks after TCRL.

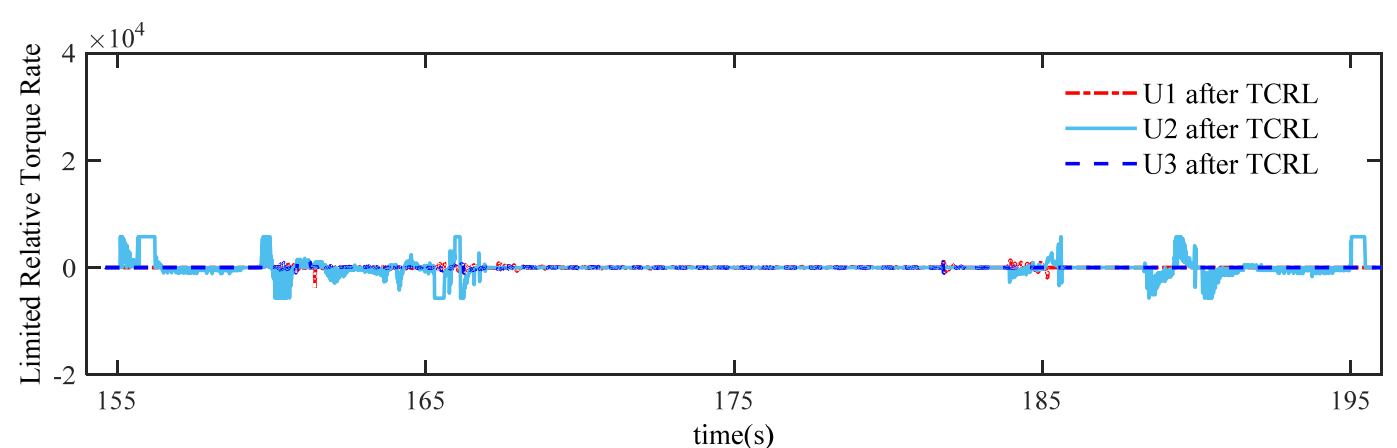

(a)

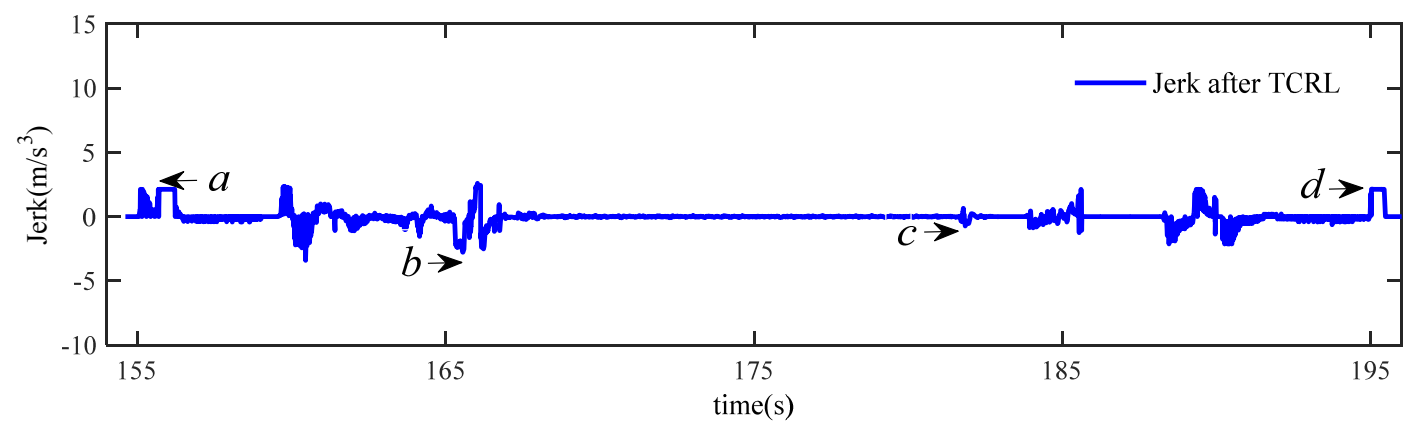

(b)

Figure 13. (a) Relative torque changing rates after torque changing rate limitation (TCRL) of Cycle 1 Part 4; (b) System jerk after TCRL of Cycle 1 Part 4.

The intensities of the peak jerks in Cycle 1 Part 4 before and after TCRL are summarized in Table 4. Four peak-jerk points whose initial jerk intensities exceed $5 \mathrm{~m} / \mathrm{s}^{3}$ are all controlled within $2.5 \mathrm{~m} / \mathrm{s}^{3}$ after TCRL. Quantitatively, the TCRL strategy reduces vehicle jerk by a maximum of $85.82 \%$. Therefore, the jerk occurring in real driving conditions of the investigated power-split HEB is effectively controlled, and riding comfort of it is significantly improved. Additionally, the proposed TCRL strategy limits the torque changing rates of the power sources with only a small modification of the original control strategy and is a highly-efficient anti-jerk method to smooth vehicle jerks occurring in HEVs' real driving conditions. 
Table 4. Intensities of peak jerks of Cycle 1 Part 4 before and after TCRL.

\begin{tabular}{cccc}
\hline Peak-Jerk Number & Jerk before TCRL $\left(\mathbf{m} / \mathbf{s}^{\mathbf{3}}\right)$ & Jerk after TCRL $\left(\mathbf{m} / \mathbf{s}^{\mathbf{3}}\right)$ & Jerk Reduction $\mathbf{( \% )}$ \\
\hline$a$ & 6.851 & 2.129 & 68.92 \\
$b$ & -5.806 & -2.365 & 59.27 \\
$c$ & -5.133 & -0.728 & 85.82 \\
$d$ & 11.99 & 2.133 & 82.21 \\
\hline
\end{tabular}

The proposed TCRL strategy aims to suppress vehicle jerks occurring in real driving conditions without significantly affecting vehicle performance. Therefore, the performance of the investigated power-split HEB after TCRL is verified through a comparison between the actual vehicle velocity $v_{a}$ and the model-calculated theoretical velocity after TCRL of Cycle 1 Part 4. Comparison results are displayed in Figure 14.

As shown in Figure 14, the theoretical velocity after TCRL can still follow the actual vehicle velocity with some insignificant inconsistencies, and the two theoretical velocities (one before and one after TCRL) calculated by the vehicle dynamics model are nearly identical. Thus, the proposed TCRL strategy does not significantly affect vehicle performance while improving vehicle riding comfort.

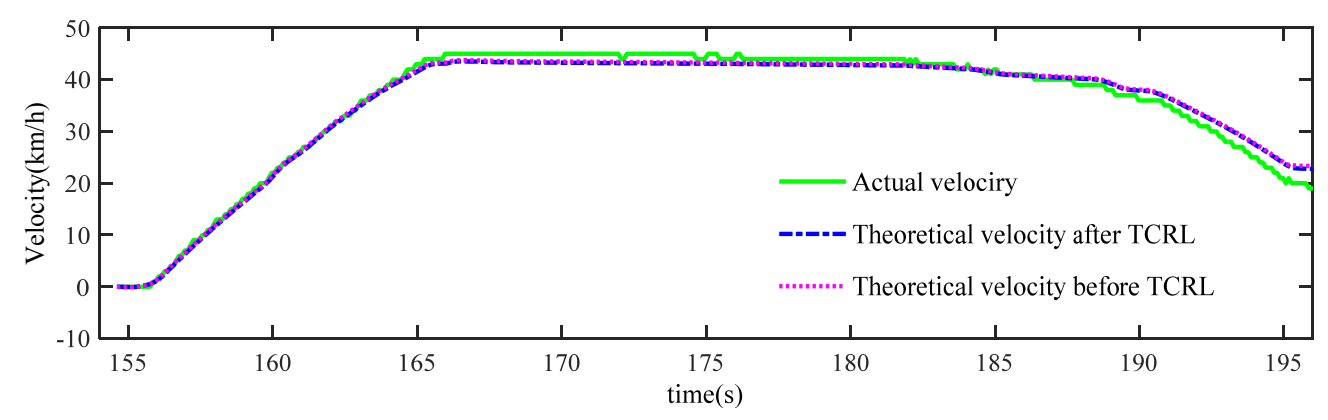

Figure 14. Velocity comparison of Cycle 1 Part 4.

The proposed TCRL control strategy can effectively suppress vehicle jerks occurring in real driving conditions of the dual planetary power-split HEB, reducing jerk intensity by a maximum of $85.82 \%$. While significantly improving vehicle riding comfort, the TCRL control strategy does not considerably compromise vehicle performance. In addition, the proposed control strategy is simple, straightforward, and easily applied to real vehicles with only a small modification of the original control strategy.

\section{Conclusions}

This study discusses the longitudinal jerk issues occurring in real driving conditions of dual planetary power-split HEBs. Focused on the coupling problem of power-source dynamic response and parameter estimation, especially dynamic torque estimation in power-split HEV jerk research, corresponding countermeasures are presented in this study. A real-vehicle-data-driven vehicle dynamics model integrating a full-time AEDM is established, and a practical anti-jerk strategy based on TCRL is proposed to suppress vehicle jerks and improve vehicle riding comfort. Results show the following:

(1) With use of engine operating data acquired from real drive cycles, a full-time AEDM with emphasis on engine starting and stopping characteristics is developed. This full-time AEDM can fully predict the real-time engine dynamic torque over an arbitrary driving cycle. On the basis of the full-time AEDM, a high-fidelity data-driven vehicle dynamics model is established. This model has sufficient accuracy to replicate vehicle jerks occurring in real driving conditions. 
(2) A detailed jerk analysis reveals that sharp changes in power source torques is the main reason for intense jerks occurring in mode transition processes in real driving conditions. A practical control strategy based on power source TCRL is proposed with the premise of not compromising vehicle performance. Verification research indicates that the TCRL strategy can reduce the original vehicle jerk by a maximum of $85.82 \%$. Thus, the riding comfort of the investigated power-split HEB is significantly improved.

In summary, this study can be used as a reference for precise vehicle dynamics modeling and riding comfort improvement of power-split HEVs and conforms to promoting the application of such system.

Author Contributions: Data curation, H.C. and Y.W.; Methodology, X.Z., D.S. and N.Y.; Software, H.C. and N.Y.; Validation, H.C.; Writing_-original draft, X.Z. and H.C.; Writing-review \& editing, T.L. and Y.L.

Acknowledgments: The authors gratefully acknowledge the National Natural Science Foundation of China (Grant No. 51575221, 51675214), the Graduate Innovation Research Project of Jilin University (Grant No. 2016083), and the Key Research and Development Plan of Shandong Province, China (No. 2017CXGC0510).

Conflicts of Interest: The authors declare no conflict of interest.

\section{References}

1. Jayakumar, A.; Chalmers, A.; Lie, T.T. Review of prospects for adoption of fuel cell electric vehicles in New Zealand. IET Electr. Syst. Transp. 2017, 7, 259-266. [CrossRef]

2. Hutchinson, T.; Burgess, S.; Herrmann, G. Current hybrid-electric powertrain architectures: Applying empirical design data to life cycle assessment and whole-life cost analysis. Appl. Energy 2014, 119, 314-329. [CrossRef]

3. Xiong, W.W.; Zhang, Y.; Yin, C.L. Optimal energy management for a series-parallel hybrid electric bus. Energy Convers. Manag. 2009, 50, 1730-1738. [CrossRef]

4. Zeng, X.; Yang, N.; Song, D.; Zhang, C.; Wang, J.; Wang, J.; Wang, Q. Multi-factor integrated parametric design of power-split hybrid electric bus. J. Clean. Prod. 2016, 115, 88-100. [CrossRef]

5. Peng, B.B.; Fan, Y.; Xu, J.H. Integrated assessment of energy efficiency technologies and $\mathrm{CO}_{2}$ abatement cost curves in China's road passenger car sector. Energy Convers. Manag. 2016, 109, 195-212. [CrossRef]

6. Finesso, R.; Spessa, E.; Venditti, M. Robust equivalent consumption-based controllers for a dual-mode diesel parallel HEV. Energy Convers. Manag. 2016, 127, 124-139. [CrossRef]

7. Cacciatori, E.; Vaughan, N.D.; Marco, J. Evaluating the Impact of drivability requirements on the performance of an energy management control architecture for a hybrid electric vehicle. In Proceedings of the 2nd IEE Conference on Automotive Electronics, London, UK, 20-21 March 2006; Institution of Electrical Engineers: Stevenage, UK, 2006; pp. 297-320.

8. Liu, D.; Zhang, J.; Zhang, D.; Liu, G.; Yu, H. Experimental and numerical analysis of the seat track vibrations caused by engine starts in a power-split hybrid electric vehicle. J. Automob. Eng. 2017, 231, 395-404. [CrossRef]

9. Yoshioka, T.; Sugita, H. Noise and vibration reduction technology in hybrid vehicle development. SAE Tech. Pap. 2001. [CrossRef]

10. Zou, L.; Zhang, J.; Yu, H. Vibration and acoustic investigation for a deep hybrid transmission with power-split compound planetary gear set. J. Mech. Eng. Sci. 2016, 230, 1331-1349. [CrossRef]

11. Tong, Y.; Ouyang, M.; Zhang, J. Real-time simulation and research on control algorithm of parallel hybrid electric vehicle. Chin. J. Mech. Eng. 2003, 39, 156-161. [CrossRef]

12. Koprubasi, K.; Westervelt, E.R.; Rizzoni, G. Toward the systematic design of controllers for smooth hybrid electric vehicle mode changes. In Proceedings of the American Control Conference, New York, NY, USA, 9-13 July 2007; pp. 2985-2990.

13. Beck, R.; Richert, F.; Bollig, A.; Abel, D.; Saenger, S.; Neil, K.; Scholt, T.; Noreikat, K.-E. Model predictive control of a parallel hybrid vehicle drivetrain. In Proceedings of the 44th IEEE Conference on Decision and Control, Seville, Spain, 15 December 2005; pp. 2670-2675.

14. Gao, B.; Liang, Q.; Xiang, Y.; Guo, L.; Chen, H. Gear ratio optimization and shift control of 2-speed I-AMT in electric vehicle. Mech. Syst. Signal Process. 2015, 50, 615-631. [CrossRef] 
15. Moriya, K.; Ito, Y.; Inaguma, Y.; Sato, E. Design of the surge control method for the electric vehicle powertrain. SAE Tech. Pap. 2002. [CrossRef]

16. Du, B.; Qin, D.; Yang, Y. Coordinated control strategies for a full hybrid electric vehicle with single motor during mode changes. Int. J. Electr. Hybrid Veh. 2016, 8, 19-40. [CrossRef]

17. Wang, C.; Zhao, Z.; Zhang, T.; Li, M. Mode transition coordinated control for a compound power-split hybrid car. Mech. Syst. Signal Process. 2017, 87, 192-205. [CrossRef]

18. Zhu, F.; Chen, L.; Yin, C.; Shu, J. Dynamic modelling and systematic control during the mode transition for a multi-mode hybrid electric vehicle. J. Automob. Eng. 2013, 227, 1007-1023. [CrossRef]

19. Zeng, X.; Yang, N.; Wang, J.; Song, D.; Zhang, N.; Shang, M.; Liu, J. Predictive-model-based dynamic coordination control strategy for power-split hybrid electric bus. Mech. Syst. Signal Process. 2015, 60, 785-798. [CrossRef]

20. Minh, V.T.; Rashid, A.A. Modeling and model predictive control for hybrid electric vehicles. Int. J. Automot. Technol. 2012, 13, 477-485. [CrossRef]

21. Wang, Y.; Yang, D.; Zhang, X.; Chen, Z. Probability based remaining capacity estimation using data-driven and neural network model. J. Power Sources 2016, 315, 199-208. [CrossRef]

22. Zhang, Y.; Guo, B. Online capacity estimation of lithium-ion batteries based on novel feature extraction and adaptive multi-kernel relevance vector machine. Energies 2015, 8, 12439-12457. [CrossRef]

23. Sun, J.; Xing, G.; Zhang, C. Data-Driven Predictive Torque Coordination Control during Mode Transition Process of Hybrid Electric Vehicles. Energies 2017, 10, 441. [CrossRef]

24. Hong, J.; Gao, B.; Lv, L.; Hu, Y.; Chen, H. Engine Speed Control During Gear Shifting of AMT HEVs with Identified Intake-to-Power Delay. IFAC-PapersOnLine 2016, 49, 793-799. [CrossRef]

25. Zhang, Y.; Luo, Q.; Ma, X.; Gong, Z. Research Method of the Dynamic Characteristics of Diesel Engine. J. Acad. Armored Force Eng. 2006, 6, 32-35. [CrossRef]

26. Yunbing, Y.; Huaming, C.; Guangde, Z. Estimation of engine torque for parallel hybrid electric vehicle. Automot. Eng. 2008, 30, 117-120. [CrossRef]

27. Yi, T. Study on the Coordinated Control Issue in Parallel Hybrid Electric System; Library of Tsinghua University: Beijing, China, 2004.

28. Arcaklioğlu, E.; Çelıkten, İ. A diesel engine's performance and exhaust emissions. Appl. Energy 2005, 80, 11-22. [CrossRef]

29. Cay, Y. Prediction of a gasoline engine performance with artificial neural network. Fuel 2013, 111, $324-331$. [CrossRef]

30. Ge, Y.; Huang, Y.; Hao, D.; Li, G.; Li, H. An indicated torque estimation method based on the Elman neural network for a turbocharged diesel engine. J. Automob. Eng. 2016, 230, 1299-1313. [CrossRef]

31. Hu, L.; Zhang, G.; Yan, Y.; Chen, H. Engine Dynamic Torque Estimation for Parallel Hybrid Electric Vehicle Based on Neural Network. Auto Mob. Sci. Technol. 2007, 4, 11-14. [CrossRef]

32. Xiang, C.; Huang, K.; Ma, Y.; Zhao, Y. Stability analysis for mode switch of multi-mode electro-mechanical transmission. In Proceedings of the IEEE Conference and Expo Transportation Electrification Asia-Pacific (ITEC Asia-Pacific), Beijing, China, 31 August-3 September 2014; pp. 1-7.

33. Park, T.; Kim, H.; Kim, J.; Han, K.; Hong, J.; Lee, H. Powertrain modeling for analyzing the transient response of the parallel HEV. In Proceedings of the 24th International Battery, Hybrid and Fuel Cell Electric Vehicle Symposium and Exhibition (EVS 24), Stavanger, Norway, 13-16 May 2009.

34. Rezaei, J.; Shahbakhti, M.; Bahri, B.; Aziz, A.A. Performance prediction of HCCI engines with oxygenated fuels using artificial neural networks. Appl. Energy 2015, 138, 460-473. [CrossRef]

35. Wang, W. Neural Network and Its Application in Automobile; Beijing Institute of Technology Press: Beijing, China, 1998; pp. 1-5.

36. Guo, L.; Ge, A.; Zhang, T.; Yue, Y. AMT shift process control. Trans. Chin. Soc. Agric. Mach. 2003, 34, 1-3. [CrossRef]

(c) 2018 by the authors. Licensee MDPI, Basel, Switzerland. This article is an open access article distributed under the terms and conditions of the Creative Commons Attribution (CC BY) license (http:/ / creativecommons.org/licenses/by/4.0/). 